\title{
Levantamento de pesquisas brasileiras sobre o Conhecimento Matemático para o Ensino e Formação de Professores
}

\author{
Rosângela Milagres Patrono \\ Ana Cristina Ferreira
}

\begin{abstract}
Resumo: Esse artigo apresenta um levantamento de pesquisas brasileiras sobre o conhecimento matemático para o ensino, a partir do banco de teses da Coordenação de Aperfeiçoamento de Pessoal de Nível Superior e da Biblioteca Digital Brasileira de Teses e Dissertações. Ao todo, foram levantadas 33 pesquisas. Todos os estudos são qualitativos, nos quais a coleta de informações se deu, principalmente, por meio de observação (apoiada por gravações em áudio e vídeo, além de diário de campo), questionários, entrevistas e registros produzidos por participantes de cursos de extensão, disciplinas e grupos de estudo. Os resultados evidenciam lacunas na formação dos participantes em relação aos conhecimentos matemáticos para o ensino e sugerem a necessidade de atenção aos mesmos tanto nos cursos de Licenciatura em Matemática e Pedagogia, quanto em ações de formação continuada.
\end{abstract}

Palavras-chave: Educação Matemática. Formação de professores de Matemática. Conhecimento matemático para o ensino. Levantamento.

\section{Survey of Brazilian research on Mathematical Knowledge for Teaching and Teacher Education}

Rosângela Milagres Patrono

Doutoranda em Educação pela Universidade Federal de Ouro Preto (UFOP), campus Mariana. Técnica e Assuntos Educacionais no IFMG, Ouro Preto, Minas Gerais, Brasil.

http://orcid.org/0000-0003-4649-1899 \rmpatrono@gmail.com

Ana Cristina Ferreira Doutora em Educação pela Universidade Estadual de Campinas (UNICAMP).

Professora Titular da Universidade Federal de Ouro Preto (UFOP), Ouro Preto, Minas Gerais, Brasil.

http://orcid.org/0000-0003-0953-1468 anacf@ufop.edu.br

Recebido em 13/10/2020 Aceito em 09/12/2020

Publicado em 13/01/2021
Abstract: This article presents a survey of Brazilian research on mathematical knowledge for teaching, from the thesis database of the Coordination for the Improvement of Higher Education Personnel and the Brazilian Digital Library of Theses and Dissertations. In all, 33 researches were surveyed. All studies are qualitative, in which the collection of information took place mainly through observation (supported by audio and video recordings, in addition to a field diary), questionnaires, interviews and records produced by participants in extension courses, disciplines and study groups. The results show gaps in the education of participants in relation to mathematical knowledge for teaching and suggest the need to pay attention to them both in the Mathematics and Pedagogy Degree courses, as well as in continuing education actions.

Keywords: Mathematics Education. Mathematics Teacher Education. Mathematics Knowledge for Teaching. Survey.

\section{Encuesta de investigaciones brasileñas acerca del Conocimiento Matemático para la Enseñanza y la Formación de Profesores}

Resumen: Ese artículo presenta una encuesta de investigaciones brasileñas acerca del conocimiento matemático para la enseñanza, a partir del banco de tesis de la Coordinación de Perfeccionamiento del Personal de Nivel Superior y de la Biblioteca Digital Brasileña de Tesis e Disertaciones. Al todo, fueran levantadas 33 investigaciones. Todos los estudios son cualitativos. La recoleta de informaciones se dio, principalmente, por medio de observaciones (apoyada por grabaciones en audio y video, además del diario de campo), cuestionarios, entrevistas y registros 
producidos por los participantes de cursos de extensión, disciplinas y grupos de estudio. Los resultados evidencian lagunas en la formación de los participantes cuanto a los conocimientos matemáticos para la enseñanza y sugieren mayor atención a los mismos tanto en los cursos de Licenciatura en Matemáticas y Pedagogía, cuanto en acciones de formación continuada.

Palabra clave: Educación Matemática. Formación de profesores de Matemáticas. Conocimiento matemático para la enseñanza. Encuesta.

\section{Introdução}

A formação de professores em geral, e a de professores de Matemática, em particular, tem mobilizado nas últimas décadas um número significativo de agentes sociais (pesquisadores, gestores, formadores e professores). Em termos de pesquisa, passamos de um número tímido nas décadas de 1970 e 1980 para uma produção numericamente expressiva a partir dos anos 2000 (FIORENTINI, 1994; FERREIRA, 2003; FIORENTINI et al., 2016). Paralelamente, observamse alguns avanços na legislação que normatiza os cursos de licenciatura, particularmente entre 2002 e 2015. Gradativamente, tais agentes sociais começam a perceber que a docência tem especificidades e que o conhecimento profissional produzido no âmbito da formação inicial necessita profunda atenção e estudo.

Contudo, tal preocupação é bem recente.

Da criação do primeiro curso de Matemática no Brasil (na USP em 1934) até pouco mais de uma década atrás, a formação do professor de Matemática era majoritariamente concebida como uma soma de conhecimento da matéria (i.e., Matemática) com conhecimento acerca do ensino, visto como transmissão de conhecimentos a outros. Nessa perspectiva, a licenciatura oferecia ao futuro professor, fundamentalmente, conhecimentos relativos à disciplina (Matemática) e conhecimentos relativos às técnicas gerais de ensino (Didática), geralmente, de forma dicotômica e com uma grande ênfase nos primeiros em detrimento dos últimos.

Tal situação espelhava um cenário existente em vários países nos quais a formação do professor de Matemática se estruturava em três anos de Matemática mais um ano de didática. Não se questionava, nem no Brasil nem no exterior, se o tipo de conhecimento matemático valorizado e desenvolvido ao longo da formação inicial era efetivamente relevante para a prática docente na escola básica (MOREIRA e FERREIRA, 2013). Em parte, isso se devia ao fato de que a Matemática era entendida (e ainda o é, em muitos espaços, inclusive acadêmicos) como única. Contudo, discussões acerca de distintas matemáticas (matemática escolar, matemática acadêmica, matemática de determinado grupo social, etc.) já vinham questionando esse status há 
décadas e, gradativamente, começaram a ganhar espaço na literatura científica na área de Educação Matemática.

Um marco nessa história surge em 1986, Lee Shulman. Reagindo, por um lado, a um tipo de pedagogismo que colocava em segundo plano as especificidades das diferentes disciplinas escolares e, por outro, a um aforismo que retratava a avaliação negativa vigente em vários segmentos da sociedade sobre o saber do professor (quem sabe faz, quem não sabe ensina), Shulman (1986, 1987), busca descrever o que chamou de Repertório de Conhecimentos para 0 Ensino (Knowledge Base for Teaching), o qual se constituiria a partir de um amplo leque de conhecimentos, superando em muito a antiga tradição do bacharelado + didática (ou o já referido 3+1) (MOREIRA e FERREIRA, 2013, p. 996).

Esse repertório, que envolvia várias categorias de saberes, se contrapunha a formas reducionistas de perceber o saber profissional do professor que, por caminhos distintos, desvalorizavam a própria profissão. "Ao final de seu artigo de 1986, sob o título Those Who Understand: Knowledge Growth in Teaching, Shulman sintetiza sua resposta, também em forma de aforismo: quem sabe faz; quem compreende ensina. A inferência é clara: para ensinar é preciso compreender, o que supõe mais que saber" (MOREIRA e FERREIRA, 2013, p. 997).

Para Moreira e Ferreira (2013), uma das categorias do Repertório de Shulman - o Conhecimento Pedagógico do Conteúdo (PCK - Pedagogical Content Knowledge) - acabou constituindo uma das mais importantes contribuições para se pensar sobre o lugar da Matemática na licenciatura, influenciando estudos desenvolvidos em várias partes do mundo, até hoje, inclusive.

A nosso ver, esse elemento do saber docente repercutiu de maneira profunda nos estudos posteriores vinculados à formação e aos saberes da profissão docente, especialmente por duas de suas características fundamentais. Em primeiro lugar, traduzia a ideia de que o professor possui um modo próprio de lidar com a Matemática, em sua prática profissional, e isso constituiu uma contribuição importante na eventual construção de alternativas teóricas consistentes para a polarização entre pedagogismo e conteudismo. Além disso, essa noção de Shulman trazia para o debate uma visão positiva das potencialidades da prática docente escolar como produtora de saber profissional, predominantemente concebido, até então, como produzido em instâncias acadêmicas e, no máximo, levado à prática pelos professores bem formados (ex. BEDNARZ e PROULX, 2009; MOREIRA e FERREIRA, 2013). 
Paralelamente, outros pesquisadores, em distintas partes do mundo, dão continuidade a esses estudos (ex. BROMME, 1994; CUOCO, 2001; SCHIFTER, 1998), argumentando, dentre outras coisas, que o professor precisa saber mais do que vai ensinar; que deve possuir uma ampla cultura matemática, que lhe permita transmitir, para além do conteúdo, valores associados ao rigor dedutivo próprio da disciplina, à precisão de linguagem etc.; é importante que o professor tenha uma visão conectada da matemática escolar, em oposição a uma visão segundo a qual a matemática se reduz a uma coleção de fórmulas e procedimentos algorítmicos esparsos e de uso ad hoc.

A noção de Mathematical Knowledge for Teaching (MKT), ou "Conhecimento Matemático para o Ensino" como tem sido usualmente traduzida no Brasil, tem sido investigada e desenvolvida por Deborah Ball e colaboradores desde o início dos anos 2000. Após mais de uma década de estudos envolvendo professores que lecionam matemática em diversos níveis de ensino, e tendo como ponto de partida os estudos pioneiros de Shulman, esse grupo de pesquisadores norteamericanos procurou detalhar os fundamentos que constituem a noção de conhecimento pedagógico do conteúdo proposta por esse autor. Nesse sentido, em uma abordagem pautada em um trabalho iniciado na prática, Ball e seus colaboradores se debruçaram sobre os conhecimentos matemáticos necessários à docência e "como" e "onde" poderiam utilizar tal conhecimento em sua ação (BALL, THAMES e PHELPS, 2008).

Outros pesquisadores, em distintas partes do mundo (ex. ADLER, 2005; BEDNARZ e PROULX, 2009) trabalharam a matemática para o ensino com novas formas de fazer dos alunos, de comunicar a Matemática em suas tarefas, de mobilizar a Matemática utilizada pelos professores. Outros exemplos da disseminação de estudos voltados para o conhecimento matemático específico do professor de Matemática podem ser encontrados nos trabalhos desenvolvidos por Davis e Renert $(2009,2014)$.

Estudos dessa natureza ou apoiados em tais referenciais ainda são relativamente recentes no Brasil, mas começam a ganhar expressividade.

No âmbito das pesquisas, está claro que a docência escolar em geral, e particularmente em Matemática, é um trabalho social complexo, no qual o professor, querendo ou não, atua no desenvolvimento de um processo de escolarização básica, que, por sua natureza eminentemente social, está sujeito a condicionamentos externos à escola, não escapa das lutas sociais mais gerais, das disputas em torno de interesses políticos, socioculturais e econômicos. Além disso, cada disciplina escolar tem distinções fundamentais em relação às demais que demandam a construção de conhecimentos próprios da docência em cada área. 
Essas breves considerações, fundamentadas por todo um corpo de evidências científicas, justificam a necessidade de se pensar a formação e desenvolvimento profissional de professores de Matemática como um lugar no qual se analisem e se vivenciem práticas de formação que envolvam os saberes específicos da docência em Matemática.

Contudo, estudos recentes indicam que, no âmbito dos cursos de Licenciatura em Matemática brasileiros, pouco se avançou nesse sentido. Ainda que programas como o PIBID1 (Programa Institucional de Iniciação à Docência) e, mais recentemente, a Residência Pedagógica ${ }^{2}$ tenham conseguido, em muitos casos, estabelecer vínculos mais estreitos com a realidade das escolas e das salas de aula de Matemática, e que a legislação (BRASIL, 2003, 2015) também determine que as matrizes curriculares dos cursos de licenciatura devam construir pontes com a escola básica, pouco se alterou efetivamente na forma como a Matemática é compreendida e desenvolvida por docentes e futuros professores no âmbito desses cursos (GATTI et al., 2019). Ainda se defende uma 'formação sólida em Matemática' para o futuro professor sem que, na maioria das vezes, se explicite o que efetivamente constituiria essa tal solidez e, menos ainda, se desenvolvam ações em consonância com tal formação sólida na prática profissional do professor de Matemática.

No presente artigo, nos propomos a realizar um levantamento da produção brasileira com foco no conhecimento matemático para o ensino, tomando como referência o Catálogo de Teses e Dissertações da Coordenação de Aperfeiçoamento de Pessoal de Nível Superior (CAPES) ${ }^{3}$ e a Biblioteca Digital Brasileira de Teses e Dissertações (BDTD) ${ }^{4}$. Nosso intuito foi tentar responder à seguinte questão: 0 que tem sido produzido no Brasil acerca da noção de conhecimento matemático para o ensino?

Para isso, discorremos brevemente sobre essa noção, descrevemos os procedimentos adotados e apresentamos e discutimos os resultados do levantamento.

\footnotetext{
1 Ver mais em http://portal.mec.gov.br/pibid.

2 Ver mais em http://portal.mec.gov.br/component/tags/tag/45681.

${ }^{3}$ CAPES, missão. Disponível em: https://www.gov.br/capes/pt-br/acesso-a-informacao/institucional/historia-e-missao. Acesso em 22 de set. de 2020.

${ }^{4}$ BDTD, o que é. Disponível em: https://bdtd.ibict.br/vufind/Content/whatls. Acesso em 22 de set. de 2020.
} 


\section{O Conhecimento Matemático para o Ensino}

O modelo teórico de conhecimento matemático para o ensino foi desenvolvido por Débora Ball e seus colaboradores. Para eles, o conhecimento matemático para o ensino envolve saberes ${ }^{5}$ relevantes na realização do trabalho de ensinar Matemática (BALL, THAMES e PHELPS, 2008).

Duas categorias da base de conhecimentos para o ensino proposta por Shulman (1987) - conhecimento do conteúdo e conhecimento pedagógico de conteúdo - foram refinadas e divididas, cada uma, em outras duas.

Figura 1: Domínios do conhecimento de conteúdo para o ensino

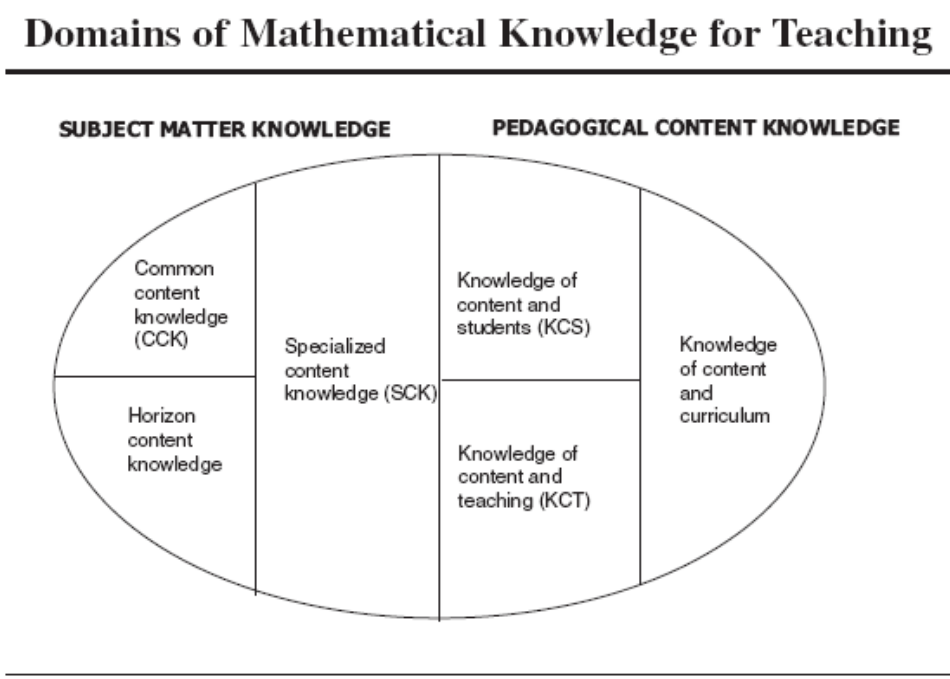

Fonte: Ball, Thames e Phelps (2008, p.403).

O conhecimento do assunto (subject matter knowledge) foi dividido em: conhecimento comum do conteúdo (common content knowledge - CCK) e conhecimento especializado do conteúdo (specialized content knowledge - SCK).

No conhecimento comum do conteúdo, o 'comum' significa que não é exclusivo do ensino, que é usado em outros cenários, que são habilidades matemáticas que outros também têm, ou seja, não é específico dos professores de Matemática.

Já o conhecimento especializado do conteúdo, os autores definem como conhecimento que é próprio do ensino, do professor de Matemática. Segundo eles "é o conhecimento e a habilidade matemática exclusivos do ensino [...]. Um exame minucioso revela que o SCK é um

\footnotetext{
${ }^{5}$ No presente artigo, consideraremos saberes e conhecimentos como sinônimos.
} 
conhecimento matemático que normalmente não é necessário para outros fins que não o ensino ${ }^{6 "}$ (BALL, THAMES e PHELPS, 2008, p. 400, tradução nossa).

Uma terceira categoria, conhecimento do horizonte do conteúdo (horizon content knowledge), foi incluída nesse domínio, porém os autores não têm certeza se ela é parte do conhecimento comum do conteúdo ou se pode ser trabalhada em outras categorias. Esse conhecimento refere-se ao entendimento de como os conteúdos matemáticos se relacionam no currículo.

O conhecimento pedagógico do conteúdo (pedagogical content knowledge) também foi dividido em: conhecimento do conteúdo e dos estudantes (knowledge of content and student KCS) e conhecimento do conteúdo e do ensino (knowledge of content and teaching - KCT).

O conhecimento do conteúdo e dos estudantes combina conhecimento sobre os alunos e sobre a Matemática. Assim:

Os professores devem prever o que os alunos provavelmente pensarão e o que eles acharão confuso. Ao escolher um exemplo, os professores precisam prever o que os alunos acharão interessante e motivador. Ao atribuir uma tarefa, os professores precisam antecipar o que os alunos provavelmente farão com ela e se acharão fácil ou difícil. Os professores também devem ser capazes de ouvir e interpretar o pensamento emergente e incompleto dos alunos, conforme expresso nas formas como os alunos usam a linguagem7 (BALL, THAMES e PHELPS, 2008, p .401, tradução nossa).

A execução dessas tarefas requer do professor uma interação entre a compreensão de um conteúdo matemático específico e a familiaridade com os alunos e o pensamento matemático deles.

O conhecimento do conteúdo e do ensino combina conhecimento sobre o ensino e sobre a Matemática. É a forma como os professores organizam os conteúdos para a instrução escolhem com quais exemplos vão começar, quais vão usar para aprofundar um conceito, avaliam as vantagens e desvantagens de representações usadas para ensinar um conteúdo, quais métodos e procedimentos oferecem mais possibilidade de aprendizagem. "Cada uma dessas

\footnotetext{
6 "...is the mathematical knowledge and skill unique to teaching (...). Close examination reveals that SCK is mathematical knowledge not typically needed for purposes other than teaching" (BALL, THAMES e PHELPS, 2008, p. 400).

7 "Teachers must anticipate what students are likely to think and what they will find confusing. When choosing an example, teachers need to predict what students will find interesting and motivating. When assigning a task, teachers need to anticipate what students are likely to do with it and whether they will find it easy or hard. Teachers must also be able to hear and interpret students' emerging and incomplete thinking as expressed in the ways that pupils use language" (BALL, THAMES e PHELPS, 2008, p. 401).
} 
tarefas requer uma interação entre compreensão matemática específica e compreensão de questões pedagógicas que afetam a aprendizagem do aluno"” (BALL, THAMES e PHELPS, 2008, p. 401, tradução nossa).

A categoria conhecimentos curriculares proposta por Shulman (1986) foi posicionada pelos autores nesse domínio. Novamente, eles afirmam não ter certeza se pode ser uma categoria em si, se é parte da categoria conhecimento do conteúdo e do ensino ou se pode ser trabalhada em várias categorias.

Os estudos de Ball e colaboradores influenciaram (e seguem influenciando) o desenvolvimento de pesquisas e propostas de formação em todo o mundo.

A seguir apresentamos a metodologia e os resultados encontrados.

\section{Metodologia}

Metodologicamente, entendemos o mapeamento da pesquisa conforme Fiorentini et al (2016, p. 18) "como um processo sistemático de levantamento e descrição de informações acerca das pesquisas produzidas sobre um campo específico de estudo, abrangendo um determinado espaço (lugar) e período de tempo". Para esses autores, o mapeamento da pesquisa se diferencia do estado da arte da pesquisa porque "faz referência à identificação, à localização e à descrição das pesquisas realizadas num determinado tempo, espaço e campo de conhecimento" (FIORENTINI et al, 2016, p. 18).

Dessa forma, realizamos um levantamento e descrição de pesquisas brasileiras que abordam o Conhecimento Matemático para o Ensino ou algum termo próximo (ex. matemática para o ensino de Davis e Renert, 2009, 2014). Inicialmente foi usado o Catálogo de Teses e Dissertações da CAPES ${ }^{9}$. Como o principal texto que aborda a noção de Conhecimento Matemático para o Ensino (Ball e colaboradores) é de 2008, consideramos as teses e dissertações defendidas após essa data.

Utilizamos os seguintes termos de busca: a) "Matemática para o ensino", b) "Conhecimento matemático para o ensino" e c) "Formação de professores" AND "Conhecimento matemático para o ensino".

\footnotetext{
8 "Each of these tasks requires an interaction between specific mathematical understanding and an understanding of pedagogical issues that affect student learning" (BALL, THAMES e PHELPS, 2008, p. 401).

${ }^{9}$ Escolhido por ser a principal agência oficial a reconhecer e certificar cursos de Pós-Graduação Strictu sensu no país.
} 
Na primeira busca ("Matemática para o ensino"), localizamos 115 pesquisas. Analisamos os resumos e apenas sete estudos abordavam o construto teórico foco deste levantamento ${ }^{10}$. Com o descritor "conhecimento matemático para o ensino" foram levantadas 24 pesquisas, sendo uma delas já identificada. Finalmente, na terceira busca ("formação de professores" AND "conhecimento matemático para o ensino"), foram identificadas 17 pesquisas, mas todas já haviam sido citadas nas buscas anteriores.

Procurando complementar o levantamento, consultamos a Biblioteca Digital Brasileira de Teses e Dissertações (BDTD). A busca com o termo "conhecimento matemático para o ensino" apresentou 26 pesquisas. Desse total,13 já constavam do levantamento e 10 foram excluídas porque não se referiam à noção em estudo nem envolviam seu uso na formação de professores ${ }^{11}$. Assim apenas três foram selecionadas. Em uma segunda busca ("formação de professores" AND "conhecimento matemático para o ensino") foram identificadas nove pesquisas, porém, oito já haviam sido citadas antes e uma foi excluída por não abordar a noção em estudo.

Dessa forma, foram levantadas ao todo 33 pesquisas sendo 19 dissertações e 14 teses. De acordo com o gráfico abaixo, o número maior de pesquisas defendidas foi no ano de 2015.

Figura 2: Quantidade de pesquisas por ano de defesa

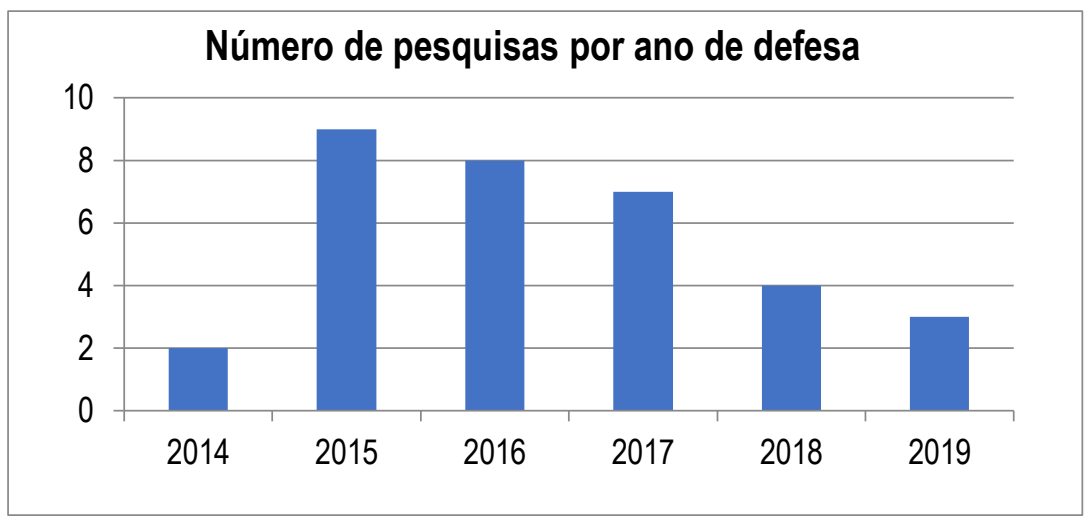

Fonte: Elaborado pelas autoras.

Mais da metade das pesquisas (22) foram defendidas em instituições da região Sudeste e a maioria no estado de São Paulo (18 pesquisas). A região Nordeste aparece em segundo com oito pesquisas e quatro delas no estado da Bahia. Duas pesquisas foram defendidas na região Sul (ambas no Paraná) e uma na região Centro-Oeste (em Mato Grosso do Sul). Não identificamos nenhum estudo na região Norte.

\footnotetext{
10 Em muitos casos, o termo era parte de um maior (matemática para o ensino fundamental...) e não se referia à noção em estudo.

${ }^{11}$ Nos estudos excluídos, o conhecimento matemático associado ao ensino estava relacionado a temáticas como olímpiada de Matemática, linguagem matemática, aprendizagem baseada em problemas, dentre outras.
} 
Figura 3: Concentração das pesquisas por estado

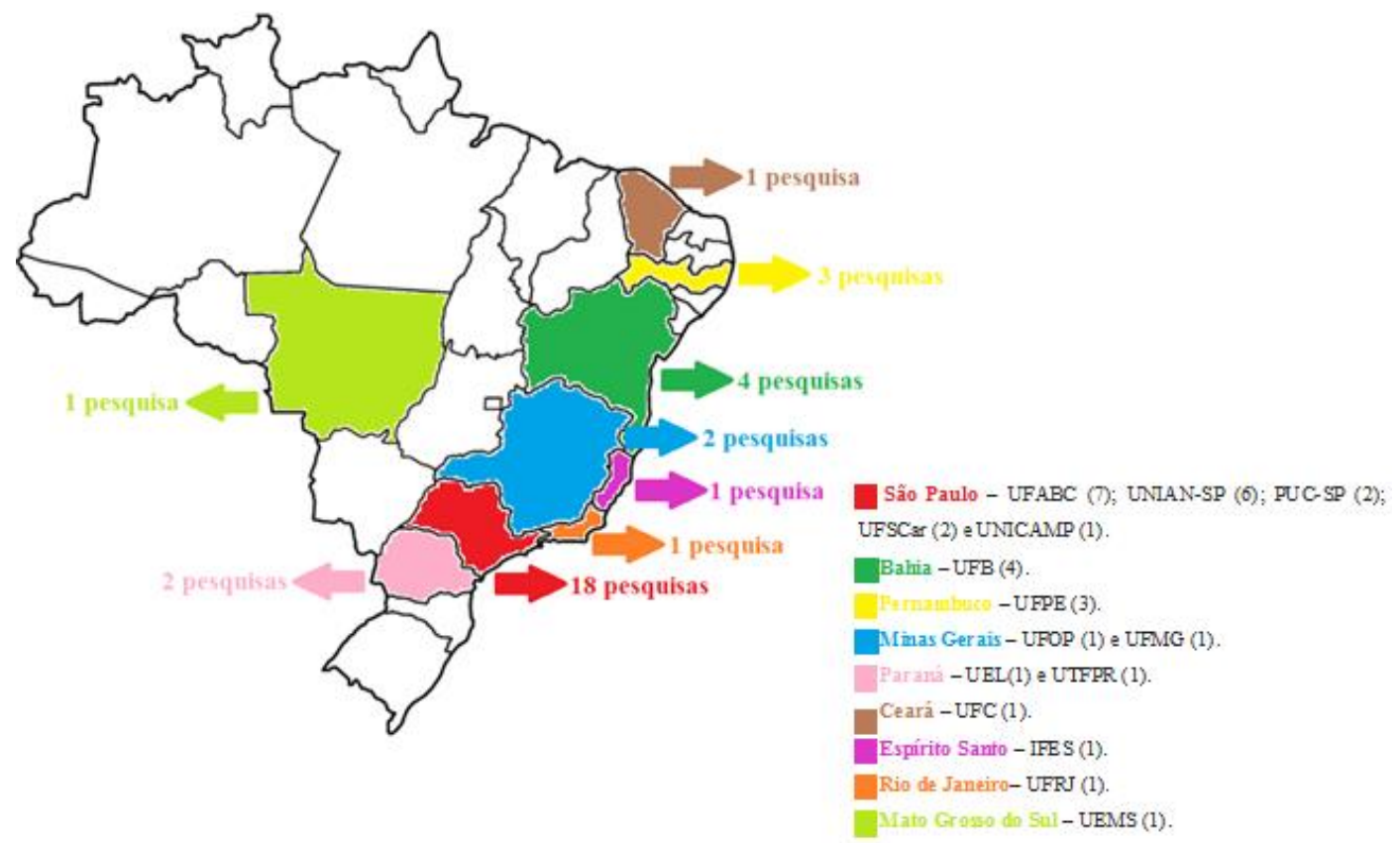

Fonte: Elaborado pelas autoras.

Vinte e um pesquisadores orientaram os trabalhos.

Tabela 1: Organização do número de pesquisas por instituição e orientadores

\begin{tabular}{|c|c|l|l|}
\hline Instituição & $\begin{array}{c}\text { Número de } \\
\text { pesquisas } \\
\text { orientadas }\end{array}$ & \multicolumn{1}{|c|}{ Orientador(a) } & \multicolumn{1}{|c|}{ Ano de defesa/nível } \\
\hline UFABC & 7 & Alessandro Jacques Ribeiro (6) & $\begin{array}{l}2014(\mathrm{D}) ; 2015(2 \mathrm{D}) ; 2016 \text { (D); } \\
2017(\mathrm{D}, \mathrm{T}) \\
2015(\mathrm{D})\end{array}$ \\
\hline UNIAN & 6 & $\begin{array}{l}\text { Runc Cesar Pietropaolo (5) } \\
\text { Angélica da Fontoura Garcia Silva (1) }\end{array}$ & $\begin{array}{l}2015(2 \mathrm{C}) ; 2016(2 \mathrm{D}) ; 2018(\mathrm{~T}) \\
2016(\mathrm{D})\end{array}$ \\
\hline UFB & 4 & Jonei Cerqueira Barbosa (4) & $2015(\mathrm{D}) ; 2016$ (T); 2017 (2T) \\
\hline UFPE & 3 & $\begin{array}{l}\text { Marcelo Câmara dos Santos (1) } \\
\text { Gilda Lisbôa Guimarães (1) }\end{array}$ & $\begin{array}{l}2015(\mathrm{D}) \\
2018(\mathrm{~T})\end{array}$ \\
& & Rosinalda Aurora de Melo Teles (1) & $2017(\mathrm{D})$ \\
\hline PUC-SP & 2 & $\begin{array}{l}\text { Cileda de Queiroz e Silva Coutinho (1) } \\
\text { Maria José Ferreira da Silva (1) }\end{array}$ & $\begin{array}{l}2019(\mathrm{D}) \\
2015(\mathrm{~T})\end{array}$ \\
\hline UFSCar & 2 & $\begin{array}{l}\text { Ademir Donizeti Caldeira (1) } \\
\text { Bárbara Cristina M. S. Nakayama (1) }\end{array}$ & $\begin{array}{l}2016(\mathrm{~T}) \\
2018(\mathrm{D})\end{array}$ \\
\hline UFOP & 1 & Ana Cristina Ferreira & $2017(\mathrm{D})$ \\
\hline UNICAMP & 1 & Dario Fiorentini & $2015(\mathrm{~T})$ \\
\hline UFMG & 1 & Maria Manuela Martins Soares David & $2014(\mathrm{~T})$ \\
\hline UEMS & 1 & Antônio Sales & $2016(\mathrm{D})$ \\
\hline UFC & 1 & José Aires de Castro Filho & $2017(\mathrm{~T})$ \\
\hline UFL & 1 & Ângela Marta Pereira das Dores Savioli & $2019(\mathrm{~T})$ \\
\hline UTFPR & 1 & Jader Otávio Dalto & $2019(\mathrm{D})$ \\
\hline IFES & 1 & Maria Auxiliadora Vilela Paiva & $2018(\mathrm{D})$ \\
\hline UFRJ & 1 & Victor Augusto Giraldo & $2016(\mathrm{D})$ \\
\hline
\end{tabular}

Fonte: Elaborado pelas autoras (Obs. D - Dissertação, T - Tese) 
Observamos que os professores Alessandro Jacques Ribeiro, Ruy Cesar Pietropaolo e Jonei Cerqueira Barbosa orientaram 15 dos 33 estudos. Todos os demais professores orientaram um trabalho cada.

As pesquisas orientadas por Ruy César Pietropaolo (duas dissertações e três teses) se desenvolveram a partir do mesmo percurso metodológico: o Design Experiments (MACEDO, 2016; MARQUES, 2018; MATEUS, 2015; ROGERI, 2015; SERA, 2016). De acordo com Cobb et al (apud ROGERI, 2015, p. 47), o "Design Experiments possui uma natureza intervencionista, com características prospectivas e reflexivas, que possibilitam melhorar o design inicial por meio de testes e revisões de conjecturas, prevendo ciclos interativos de design, aplicação, análise e redesign". Essa metodologia envolve duas fases e os autores utilizaram na primeira fase instrumentos, questionários, grupo focal. Na segunda fase aconteceram os processos formativos segundo o Design Experiments.

Jonei Cerqueira Barbosa orientou três teses e uma dissertação do corpus em análise. Todas foram estruturadas no formato multipaper12 (BORTOLOTI, 2016; COUTINHO, 2015; GOMEZ, 2017; SANTOS, 2017).

Os seis estudos (cinco dissertações e uma tese) orientados por Alessandro Jacques Ribeiro da UFABC abordam temas relacionados ao ensino de álgebra (simetria na álgebra escolar, pensamento algébrico, equações, polinômios), mas a metodologia foi diferenciada - questionários e análise de documentos de aulas preparadas sobre equações por seis professores (OLIVEIRA, 2014); questionário, entrevistas e grupo focal onde se discutiu o conceito de equação (SILVA, 2015); análise documental (documentos oficiais e manuais do professor) buscando compreender como o conceito de simetria se manifesta na álgebra (OLIVEIRA, 2015); análise interpretativa de dados coletados em um curso de extensão sobre o pensamento algébrico nos anos iniciais do Ensino Fundamental (formato multipaper) (FERREIRA, 2017); questionário aplicado a licenciandos de quatro universidades buscando identificar e compreender as concepções sobre equações (ALMEIDA, 2016); análise de questionários e registros escritos, elaborados/produzidos pelos professores-participantes de um curso de extensão sobre polinômios (LAUTENSCHLAGER, 2017).

No próximo tópico apresentamos uma análise e descrição dessas pesquisas.

\footnotetext{
12 Nesse formato, no primeiro capítulo, os autores descrevem a trajetória até chegar no problema de pesquisa, apresentam a justificativa, o referencial, os objetivos e o formato da dissertação ou tese. Os outros capítulos são artigos independentes publicados que tratam o problema de formas diferentes e, às vezes são escritos no idioma exigido pelo periódico no qual se planeja submeter o texto.
} 


\section{Resultados}

Ainda que a maioria das pesquisas tenha sido desenvolvida com professores da Educação Básica (22), existem algumas que consideraram outras possibilidades. Dessa forma, organizamos o corpus em cinco categorias tendo como parâmetro os participantes de cada estudo:

Tabela 2: número de pesquisas por categoria

\begin{tabular}{|l|c|}
\hline \multicolumn{1}{|c|}{ Categoria } & Número de pesquisas \\
\hline Pesquisas com professores da Educação Básica & 22 \\
\hline Pesquisas com licenciandos & 6 \\
\hline Pesquisas com licenciandos e professores & 2 \\
\hline Pesquisa documental ou bibliográfica & 2 \\
\hline Pesquisa de si & 1 \\
\hline
\end{tabular}

Fonte: Elaborado pelas autoras.

Apresentamos a seguir uma breve descrição dos estudos em cada categoria e finalizamos com algumas reflexões sobre o corpus como um todo.

\subsection{Pesquisas com professores da Educação Básica}

Nessa categoria encontram-se 22 pesquisas. Em uma os participantes são professores da Educação Infantil (MOURA, 2016), em seis professores que atuam nos Anos Iniciais do Ensino Fundamental (FILHO, 2015; OLIVEIRA, 2015; RIBEIRO, 2016; ROGERI, 2015; SILVA S., 2016; SILVA, 2017) e em quinze professores que atuam no Ensino Fundamental II e Ensino Médio.

Os estudos se fundamentam no MKT (BALL, THAMES e PHELPS, 2008) para investigar conhecimento matemático para o ensino de vários temas matemáticos como: área e perímetro (SILVA S., 2016), números racionais (FILHO, 2015; MARQUES, 2018; ROGERI, 2015; SOUZA, 2015), educação financeira (MARTINS; 2019), escala (ALBUQUERQUE, 2018), álgebra (equações (ALMEIDA, 2016; OLIVEIRA, 2014; SILVA, 2015), simetria (OLIVEIRA, 2015), pensamento algébrico (FERREIRA, 2017), polinômios (LAUTENSCHLAGER, 2017), conceito de variável (GOMEZ, 2017), conceito de função (SANTOS, 2017)), proporcionalidade (BORTOLOTI, 2016), combinação simples (COUTINHO, 2015), estatística (MACEDO, 2016), leitura e construção de gráficos (SERA, 2016), figuras geométricas (SILVA, 2017).

Em alguns estudos o foco maior não foi o tema matemático. Silva C. (2016) aplicou e analisou uma tarefa desenhada com 0 intuito de explorar o conhecimento matemático para 0 ensino com um grupo de professores mestrandos por meio de suas próprias reflexões. A atividade da tarefa envolveu a divisão de números naturais em uma base não decimal. Serra (2018) se propôs a compreender os conhecimentos matemáticos para o ensino mobilizados por professores 
da Educação Básica quando refletiam sobre os "porquês" dos alunos. Ribeiro (2016) trabalhou com professores dos anos iniciais a Modelagem Matemática e a mobilização de conhecimentos didáticos matemáticos usando o MKT (BALL, THAMES e PHELPS, 2008) e o modelo do Conhecimento Didático-Matemático (GODINO, 2009 apud RIBEIRO, 2016). No problema proposto, durante as estratégias utilizadas para busca de solução, foi discutido o trabalho que os professores realizam em sala de aula em torno dos quadriláteros. Moura (2016) investigou que conhecimentos o profissional docente da Educação Infantil expressa em suas práticas e discursos em relação ao ensino da Matemática.

De modo geral, todos os 22 estudos apresentaram uma discussão do MKT (BALL, THAMES e PHELPS, 2008) e outros referenciais de acordo com a temática abordada.

Os procedimentos para a coleta de informações e produção de dados envolveram: questionários (às vezes, combinado com outros instrumentos) (FILHO, 2015; LAUTENSCHLAGER, 2017; MACEDO, 2016; OLIVEIRA, 2014; ROGERI, 2015; SERA, 2016; SOUZA, 2015), registros produzidos pelos professores nos cursos de extensão (BORTOLOTI, 2016; COUTINHO, 2015; FERREIRA, 2017; GOMEZ, 2017; SANTOS, 2017), observações de aulas (FERREIRA, 2014; MOURA, 2016), grupo focal (SERRA, 2018; SILVA, 2015), gravações de processo de formação (ALBUQUERQUE, 2018; SILVA, 2017), análise de planos de aula (MARTINS, 2019), análise de uma tarefa (SILVA C., 2016), registros produzidos em um grupo de estudo (SILVA S., 2016); gravação de encontros de formação (RIBEIRO, 2016).

De uma maneira geral, os resultados mostraram que os professores que participaram de cursos de extensão, encontros de formação, processo de formação, grupo de estudo ampliaram seus conhecimentos matemáticos para o ensino mobilizando conhecimentos profissionais relativos a variados domínios do MKT. Silva S. (2016, p. 111) observou que "depois da participação do processo formativo, depois das vivências e estudos realizados pelo grupo, as professoras adquiriram maior segurança até para analisar o conteúdo presente no livro didático".

Já Rogeri $(2015$, p. 258) afirma que:

As reflexões compartilhadas do grupo - docentes, formadora e pesquisadores - sobre as sequências de atividades, leituras e tarefas propostas, envolvendo diferentes estratégias, abordagens e contextos, além de terem provocado conflitos de ideias, aparentemente, compreendidas, promoveram a revisão de conceitos e propriedades, de modo a consolidar, ou mesmo ressignificar esses conhecimentos.

Alguns resultados mostraram também fragilidades na formação, fragilidades dos professores nos conteúdos abordados e a necessidade de promoção de ações que possam 
ampliar, aprofundar o conhecimento específico do professor de Matemática. Filho (2015) investigou como professores dos anos iniciais (Ensino Fundamental I) avaliam propostas de ensino para o trabalho com números racionais e encontrou que:

Os resultados apresentados evidenciam a fragilidade do conhecimento desses docentes em relação aos números racionais e como isto pode influenciar a sua prática pedagógica, uma vez que propostas de ensino consideradas erradas na análise prévia do questionário eles julgaram corretas e usariam ou poderiam usá-las em sala de aula (FILHO, 2015, p. 124).

Os resultados desses trabalhos realizados com professores reforçam a importância de se proporcionar oportunidades de desenvolvimento profissional para os professores que ensinam Matemática, principalmente, no que se refere aos conhecimentos próprios da profissão e "ações formativas que estimulem o desenvolvimento dos conhecimentos dos subdomínios propostos por Ball, Thames e Phelps podem potencializar os docentes a tomar decisões coerentes com suas intenções no trabalho [...]" (MARTINS, 2019, p. 103).

\subsection{Pesquisas com licenciandos}

Os seis estudos reunidos nesta categoria foram realizados com licenciandos em Matemática (ALMEIDA, 2016; CADE, 2018; CARVALHO R., 2017; MARQUES, 2018; MATEUS, 2015) e em Pedagogia (CARVALHO H., 2017). O trabalho desenvolvido ocorreu em disciplinas do curso (CADE, 2018; CARVALHO H., 2017), em processos formativos ${ }^{13}$ (MARQUES, 2018; MATEUS, 2015), por meio de pesquisa colaborativa ${ }^{14}$ (CARVALHO R., 2017) e análise de questionário aplicados a turmas de ingressantes e concluintes do curso de Licenciatura em Matemática de quatro universidades em horário de aulas (ALMEIDA, 2016).

Os propósitos das pesquisas variaram entre analisar (possíveis mobilização de saberes, o processo de construção de conceitos), identificar (e compreender as concepções, formas de construção do conceito), investigar os conhecimentos e refletir sobre o tipo de formação inicial.

Como nas pesquisas com professores, os referenciais utilizados combinaram o MKT (BALL, THAMES e PHELPS, 2008) com a literatura referente à formação do pedagogo, conhecimento de conteúdo pedagógico tecnológico, teoria dos campos conceituais, equações, geometria, estruturas multiplicativas, números racionais.

\footnotetext{
13 Intervenções realizadas pelos pesquisadores.

14 Todos trabalham conjuntamente e apoiam-se mutuamente, visando atingir objetivos comuns (CARVALHO R., 2017).
} 
A participação oportunizou aos licenciandos a reflexão sobre formas de ensinar, a ampliação dos conhecimentos, momentos de discussão.

[...] os momentos de reflexão, fossem eles no coletivo ou em pequenos grupos, permitiram que esses futuros docentes refletissem sobre formas de ensinar o conteúdo de Equações Diofantinas Lineares (EDL), articulando o saber científico aprendido na formação inicial com a perspectiva de sala de aula, ao se colocarem como professores (CADE, 2018, p. 93).

[...] as nossas sequências e as discussões fomentadas contribuíram para ampliar não apenas o conhecimento do conteúdo e do currículo, mas também a compreensão de estratégias que podem favorecer a construção de conceitos e atitudes [...] (MATEUS, 2015, p. 173).

Para Carvalho R. (2017, p. 152), "a experiência desenvolvida mostrou que o modelo de formação colaborativa, favoreceu a ampliação do campo conceitual multiplicativo pelos participantes da pesquisa". Já Carvalho H. (2017, p. 166) comenta que o tempo foi insuficiente para mobilização de saberes específicos de geometria, mas "o processo vivido contribuiu para a construção de uma relação com a geometria por parte dos licenciandos que pode favorecer aprendizagens futuras".

Marques (2018, p. 204), destaca as fragilidades da formação inicial em relação à preparação para a prática futura dos licenciandos e afirma que "a construção de uma base de conhecimentos para o ensino deve ser o eixo central da formação de professores. Desse modo, os conteúdos de ensino da Educação Básica devem ser objetos de estudo dos cursos de formação inicial".

\subsection{Pesquisas com licenciandos e professores}

Dois estudos envolveram tanto licenciandos e professores.

Marins (2019) investigou conhecimentos matemáticos para o ensino desenvolvidos em dois processos formativos que envolviam professores e licenciandos em Matemática participantes de PIBID. Os resultados evidenciaram uma mobilização de conhecimento do conteúdo e dos estudantes, conhecimento do conteúdo e do ensino e conhecimento especializado do conteúdo nos momentos de planejamento. Segundo ela, algumas práticas letivas contribuíram para isso:

\footnotetext{
ao escolher uma tarefa desafiante e interessante aos alunos; ao antecipar suas possíveis resoluções; ao explicar a dinâmica da aula; ao usar um material manipulável; ao monitorar a realização da tarefa; ao selecionar as resoluções a serem discutidas; ao sequenciá-las a fim de propiciar um encadeamento lógico das ideias; em manter um clima harmonioso para a discussão das ideias matemáticas; e ao conectar as respostas dos alunos (MARINS, 2019, p. 211).
} 
Pereira (2019) desenvolveu um curso de extensão com professores dos anos iniciais do Ensino Fundamental e licenciandos em, buscando levantar conhecimentos matemáticos para 0 ensino elaborados ou aprendidos em Tarefas de Análise da Produção Escrita ${ }^{15}$. Para ela:

\begin{abstract}
Muitos dos domínios relacionados ao MKT são intrínsecos as experiências vividas e ações tomadas pelos professores em exercício. Assim, os possíveis aspectos que esses futuros professores apresentam acerca do MKT são oriundos de poucos e específicos momentos de prática oferecidos durante cursos de formação (PEREIRA, 2019, p. 79).
\end{abstract}

Ambas as pesquisas utilizam entrevistas, gravações em áudio e vídeo, registros dos participantes, diário de bordo das pesquisadoras para a coleta de informações e produção de dados. Além disso, também destacam a importância dos momentos de trabalhos práticos, ou voltados para a prática docente, e a reflexão sobre eles na formação do professor de Matemática.

\title{
4.4 Pesquisa documental ou bibliográfica
}

Dois estudos usaram a metodologia do estudo bibliográfico: Almeida (2015) e Oliveira (2015).

Almeida $(2015)^{16}$ procurou identificar os saberes docentes mobilizados para que sólidos arquimedianos sejam ensinados na escola básica. Sua análise pautou-se nas noções de Conhecimento Matemático para o Ensino (BALL, THAMES e PHELPS, 2008), Conhecimento Tecnológico para o Ensino (MISHRA e KOEHLER, 2006) e Teoria Antropológica do Didático (CHEVALLARD, 1999) além da literatura sobre geometria e os sólidos arquimedianos.

A partir da pesquisa bibliográfica, a autora propõe um conjunto de tarefas com os sólidos arquimedianos no ambiente de geometria dinâmica CABRI 3D. Com base nas discussões feitas, Almeida (2015, p. 180) acredita que "é o conhecimento didático tecnológico que pode dar suporte às mudanças de ênfase em atividades de ensino, porque esse conhecimento precisa considerar os três componentes, matemático, didático e tecnológico [...]".

Oliveira (2015) busca compreender como o conceito de simetria se manifesta na álgebra escolar por meio de documentos oficiais (Parâmetros Curriculares Nacionais - PCN, Proposta Curricular do Estado de São Paulo) e manuais do professor do caderno do Estado de São Paulo.

\footnotetext{
15 Um instrumento e ou atividade advindo de uma produção escrita previamente analisada pelo professor (construída com o objetivo de nortear o ensino e a aprendizagem de um conteúdo) (PEREIRA, 2019).

16 Todos os autores mencionados nesse parágrafo foram citados por Almeida (2015) em sua pesquisa.
} 
Por meio da análise de conteúdo (BARDIN, 2011 apud OLIVEIRA, 2015), discute a ocorrência das unidades de registro - UR (simetria, simetrias) encontradas. As análises evidenciaram a escassez de situações que contemplem o conceito de simetria articulado à álgebra ou nela inseridos. Finalizando, a autora conclui que "o trabalho apresenta o estudo do conceito "simetria" como um potencial ponto de interlocuções e relações entre a álgebra e a geometria" (OLIVEIRA, 2015, p. 73).

Os dois trabalhos constituem-se em referenciais importantes para auxílio à prática docente escolar desses temas e sublinha, a nosso ver, a importância da produção de materiais de apoio ao trabalho do professor.

\subsection{Pesquisa de si}

Com o propósito de compreender como as experiências de aprendizagem vem influenciando a prática de ensinar matemática, Ferrasso (2015) desenvolveu um estudo de si mediante análise de narrativa de aprendizagem - situações dialógicas com alunos, com outros professores, com a literatura sobre o ensino e com outras mídias - de sua trajetória de discente e docente.

A autora ${ }^{17}$ embasou sua pesquisa em referenciais relacionados às experiências de aprendizagem (WENGER, 2001), ao conhecimento matemático do professor (BALL, THAMES e PHELPS, 2008), às práticas formativas (LAVE, 1991; FIORENTINI, 2011), à identidade profissional (FLORES, 2010).

Os cursos de formação, atividades realizadas, algumas questões resolvidas, experiências em sala de aula vão sendo contados entrelaçados com a literatura deixando as marcas de seu desenvolvimento profissional e constituição de sua identidade profissional. Comentando sobre a participação no Grupo de Sábado, a autora afirma:

\footnotetext{
Eu conheci, durante minha participação no Grupo de Sábado, a prática de elaborar tarefas exploratório-investigativas, mediada pela discussão e produção de significado de cada palavra escolhida. Nesse processo, os professores aprendem, além do conteúdo matemático, a como explorá-los na prática de sala de aula, desenvolvendo os domínios do 'conhecimento matemático para o ensino'. [...] Esta prática me tornou uma professora com autonomia para desenvolver práticas de sala de aula. Reconheço-me membro desta comunidade quando percebo que, apesar de minha autonomia e minhas crenças pessoais, é fundamental compartilhar aspectos da tarefa docente com outros professores (FERRASSO, 2015, p. 173).
}

\footnotetext{
17 Todos os autores mencionados nesse parágrafo foram citados por Ferrasso (2015) em sua pesquisa.
} 
Os resultados revelam a influência da vida de estudante nas escolhas profissionais expressas nas crenças da professora. Também evidenciam a importância de o professor buscar espaços de formação em comunidades que valorizam a pesquisa e fazer pesquisa para se desenvolver enquanto profissional (FERRASSO, 2015).

Em síntese, em todas as categorias anteriormente descritas, o conceito de conhecimento matemático para o ensino (MKT) foi utilizado pelos autores: na compreensão da matemática para o ensino como um modelo teórico apresentado por uma estrutura teórica que organiza formas de ocorrência em torno de conceitos específicos (BORTOLOTI, 2016; COUTINHO, 2015; GOMEZ, 2017; SANTOS, 2017); como base teórica para elaboração de questionários e sequências para cursos formativos (metodologia do Design Experiments), bem como na análise das falas, reflexões e respostas dos professores e ou licenciandos participantes (MACEDO, 2016; MATEUS, 2015; MARQUES, 2018; ROGERI, 2015; SERA, 2016); como base teórica para compreender, investigar, explorar, analisar a mobilização de conhecimento matemático para o ensino, durante a preparação de aulas (MARTINS, 2019; OLIVEIRA, 2014), ao resolver e analisar questões (ALBUQUERQUE, 2018; SERRA, 2018), ao analisar uma tarefa (SILVA C., 2016), ao construir e utilizar um jogo com recurso didático (SILVA, 2017), ao analisar propostas de ensino (FILHO, 2015), ao analisar questionários e participação em processos formativos (ALMEIDA, 2016; FERREIRA, 2017; LAUTENSCHLAGER, 2017; RIBEIRO, 2016; SILVA 2015; SILVA S., 2016), ao observar aulas (FERREIRA, 2014; MOURA, 2016), ao analisar documentos (OLIVEIRA, 2015); ao apresentar uma organização matemática justificada (ALMEIDA, 2015).

Em alguns casos (ex. SOUZA, 2015), o estudo se circunscreveu a um domínio do conhecimento pedagógico do conteúdo e, em outros, foi usado também como suporte na compreensão da relação entre conhecimentos e práticas de ensinar e aprender Matemática na constituição profissional de si (ex. FERRASSO, 2015).

Em nenhum dos trabalhos analisados encontramos 0 desenvolvimento dos conhecimentos matemáticos para o ensino de um tema matemático de forma que abarcasse todas as categorias do MKT, ou seja, que proporcionasse discussões de todos os conhecimentos necessários para realizar o trabalho de ensinar Matemática (BALL, THAMES e PHELPS, 2008).

\section{Considerações Finais}

O mapeamento realizado nos trouxe uma visão do desenvolvimento dos estudos sobre 0 conhecimento matemático para o ensino no Brasil. 
Retomando nossa questão inicial: "o que tem sido produzido no Brasil acerca da noção de conhecimento matemático para o ensino?", observamos que:

- a grande maioria (30 de 33) das pesquisas foi desenvolvida com professores da Educação Básica, com licenciandos ou com ambos. Esse fato ressalta a importância desse conhecimento para a prática docente de Matemática;

- do total (30) dos estudos, apenas uma pesquisa abordou a Educação Infantil e seis pesquisaram os anos iniciais do Ensino Fundamental ( $1^{\circ}$ ao $5^{\circ}$ ano);

- a metodologia na maioria das pesquisas envolveu processos formativos como: cursos de extensão, disciplinas do curso de licenciatura, grupos de estudo, dentre outros. Em todos os casos, os resultados sugerem a ampliação da base de conhecimentos dos participantes e avanços em seu desenvolvimento profissional;

- foram discutidos os conhecimentos matemáticos para o ensino de diversos tópicos da Matemática da Educação Básica (divisão de números naturais, números racionais, pensamento algébrico, equações, polinômios, sólidos arquimedianos, figuras geométricas, área e perímetro, matemática financeira, proporcionalidade, funções, curva normal, gráficos), contudo muitos tópicos ainda não foram contemplados e mesmo os que já foram podem ser complementados com outras abordagens.

Os cursos de extensão com a participação de pesquisadores, licenciandos e professores da Educação Básica, muito presentes nos trabalhos analisados, se mostra um contexto favorável para o desenvolvimento de tarefas e para a discussão, reflexão, desenvolvimento de conhecimentos específicos da profissão docente. Além disso, podem favorecer a parceria universidade escola, articulando formação inicial e formação continuada, em uma perspectiva de desenvolvimento profissional, bem como fortalecer a tríade ensino-pesquisa-extensão (NÓVOA, 2017).

A realização desse trabalho reforçou para nós a necessidade do desenvolvimento de pesquisas que ampliem os conhecimentos específicos do professor, em particular, do professor que ensina Matemática. $O$ desenvolvimento de conhecimentos matemáticos próprios da docência dessa disciplina - considerados por Roldão (2007) como elo fraco - tanto contribui para a melhoria dos processos de ensino de aprendizagem da Matemática quanto favorece a profissionalização dos professores de Matemática. 


\section{Referências}

ADLER, Jill. Researching mathematics teacher education: The QUANTUM project and its progress. In: 13th Annual Conference of the Southern African Association for Research in Mathematics, Science and Technology Education, 2005, Windhoek. Proceedings... Windhoek: UNESCO Namibia, 2005, p. 11-24.

ALBUQUERQUE, Milka Rossana Guerra Cavalcanti de. Escala apresentada em gráficos: conhecimentos matemáticos para o ensino dos anos iniciais do ensino fundamental (crianças e EJA). 2018. 294 f. Tese (Doutorado em Educação Matemática e Tecnológica). Universidade Federal de Pernambuco, Recife, 2018.

ALMEIDA, Marieli Vanessa Rediske de. Perfil conceitual de equação: investigações acerca das concepções de alunos de licenciaturas em matemática. 2016. 204 f. Dissertação (Mestrado em Ensino e História das Ciências e da Matemática). Universidade Federal do ABC, Santo André, 2016.

ALMEIDA, Talita Carvalho Silva de. A base de conhecimento para o ensino de sólidos arquimedianos. 2015. 188 f. Tese (Doutorado em Educação Matemática). Pontifícia Universidade Católica de São Paulo, São Paulo, 2015.

BALL, Deborah Loewenberg; THAMES, Mark Hoover; PHELPS, Geoffrey Charles. Content Knowledge for Teaching: What Makes It Special? Journal of Teacher Education, 59(5) 389-407. 2008.

BEDNARZ, Nadine; PROULX, Jérômi. Knowing and using mathematics in teaching: Conceptual and epistemological clarifications. For the Learning of Mathematics, 29(3), 11-17, 2009.

BORTOLOTI, Roberta D'Angela Menduni. Um estudo sobre a matemática para o ensino do conceito de proporcionalidade. 2016. 141 f. Tese (Doutorado em Educação). Universidade Federal da Bahia, Salvador, 2016.

BRASIL. Conselho Nacional de Educação. Câmara da Educação Superior. Resolução n 3/2002. Estabelece as Diretrizes Curriculares para os cursos de Matemática. Brasília, 2003.

Conselho Nacional de Educação. Conselho Pleno. Resolução nº 2/2015. Define as Diretrizes Curriculares Nacionais para a formação inicial em nível superior (cursos de licenciatura, cursos de formação pedagógica para graduados e cursos de segunda licenciatura) e para a formação continuada. Brasília, DF: CNE, 2015.

BROMME, Rainer. Beyond subject matter: a psychological topology of teachers'professional knowledge. In: BIEHLER, R.; et al. (Ed.). Didactics of Mathematics as a scientific discipline. Dordrecht: Kluwer, 1994. p. 73-88.

CUOCO, Al. Mathematics for teaching. Notices of the AMS, 48(2), p.169-174, 2001.

CADE, Nelson Victor Lousada. Construção coletiva de uma matemática para o ensino de Equações Diofantinas Lineares na formação inicial de professores. 2018. 108 f. Dissertação (Mestrado Profissional em Educação em Ciências e Matemática). Instituto Federal de Educação, Ciência e Tecnologia do Espírito Santo, Vila Velha, 2018. 
CARVALHO, Hudney Alves Faria de. Aprendendo a ensinar geometria nos anos iniciais do ensino fundamental: um estudo com alunos de pedagogia de uma universidade federal mineira. 2017. 192 f. Dissertação (Mestrado Profissional em Educação Matemática). Universidade Federal De Ouro Preto, Ouro Preto, 2017.

CARVALHO, Rodrigo Lacerda. Contribuições do campo conceitual multiplicativo para a formação inicial de professores de Matemática com suporte das tecnologias digitais. 2017. $180 \mathrm{f}$. Tese (Doutorado em Educação). Universidade Federal do Ceará, Fortaleza, 2017.

COUTINHO, Jean Lazaro da Encarnação. Matemática para o ensino do conceito de combinação simples. 2015. 117 f. Dissertação (Mestrado em Educação). Universidade Federal da Bahia, Salvador, 2015.

DAVIS, Brent; RENERT, Moshe. Mathematisc-for-Teaching as shared dynamic participation. For the Learning of Mathematics, 29(3), 2009, 37-43.

Yor: Routledge, 2014.

The math teachers know: profound understanding of emergent mathematics. New

FERRASSO, Thais de Oliveira. Aprendizagem e constituição profissional de uma professora de matemática: um estudo de si. 2015. 184 f. Tese (Doutorado em Multiunidades em Ensino de Ciências e Matemática). Universidade Estadual de Campinas, Campinas, 2015.

FERREIRA, Ana Cristina. Metacognição e Desenvolvimento Profissional de professores de Matemática: uma experiência de trabalho colaborativo. 2003. Tese (Doutorado). Faculdade de Educação. Universidade Estadual de Campinas, São Paulo, 2003.

FERREIRA, Maria Cristina Costa. Conhecimento matemático específico para o ensino na educação básica: a álgebra na escola e na formação do professor. 2014. 184 f. Tese (Doutorado em Educação: Conhecimento e Inclusão Social). Universidade Federal de Minas Gerais, Belo Horizonte, 2014.

FERREIRA, Miriam Criez Nobrega. Álgebra nos Anos Iniciais do Ensino Fundamental: uma análise do conhecimento matemático acerca do Pensamento Algébrico. 2017. 147 f. Dissertação (Mestrado em Ensino e História das Ciências e da Matemática). Universidade Federal do ABC, Santo André, 2017.

FILHO, Josué Ferreira dos Santos. Investigando como professores dos anos iniciais julgam propostas de ensino para o trabalho com os números racionais. 2015. $131 \mathrm{f}$. Dissertação (Mestrado em Educação Matemática e Tecnológica). Universidade Federal de Pernambuco, Recife, 2015.

FIORENTINI, Dario. Rumos da Pesquisa Brasileira em Educação Matemática: o caso da produção científica em cursos de Pós-Graduação. 1994. Tese (Doutorado em Metodologia de Ensino). Faculdade de Educação. Universidade Estadual de Campinas. Campinas, 1994.

FIORENTINI, Dario; PASSOS, Cármem Lúcia Brancaglion; LIMA, Rosana Catarina Rodrigues de (Org). Mapeamento da pesquisa acadêmica sobre o professor que ensina Matemática: período 2001-2012. E-book. 2016. Disponível em: < https://www.fe.unicamp.br/pf-fe/pagina_basica/58/ebook-mapeamento-pesquisa-pem.pdf>. Acesso em 20 de agosto de 2020. 
GATTI, Bernardete Angelina; BARRETTO, Elba Siqueira de Sá; ANDRE, Marli Elisa Dalmazo Afonso de; ALMEIDA, Patrícia Cristina Albieri de. Professores do Brasil: novos cenários de formação. [S.l: s.n.], 2019.

GOMEZ, Olmar Arley. Um modelo teórico da matemática para o ensino do conceito de variável. 2017. $161 \mathrm{f}$. Tese (Doutorado em Ensino, Filosofia de História das Ciências). Universidade Federal da Bahia, Salvador, 2017.

LAUTENSCHLAGER, Etienne. Conhecimento matemático para o ensino de polinômios na educação básica. 2017. 183 f. Tese (Doutorado em Neurociência e Cognição Instituição de Ensino). Universidade Federal do ABC, São Bernardo do Campo, 2017.

MACEDO, Robson Candeias. Conhecimentos de professores de Matemática sobre o processo de ensino e de aprendizagem de noções estatísticas - curva normal. 2016. 206 f. Dissertação (Mestrado em Educação Matemática). Universidade Anhanguera de São Paulo, 2016.

MARINS, Alessandra Senes. Conhecimentos profissionais mobilizados/desenvolvidos por participantes do PIBID em práticas de ensino exploratórias de matemática. 2019. 225 f. Tese (Doutorado em Ensino de Ciências e Educação Matemática). Universidade Estadual de Londrina, Londrina, 2019.

MARQUES, Adriana Brito Aguiar. Um Estudo dos Conhecimentos de Futuros Professores para o ensino de números racionais na Educação Básica. 2018. 239 f. Tese (Doutorado em Educação Matemática). Universidade Anhanguera de São Paulo, 2018.

MARTINS, Luis Paulo. Um estudo de caso sobre o conhecimento matemático para o planejamento de aulas de educação financeira. 2019. 167 f. Dissertação (Mestrado em Educação Matemática). Pontifícia Universidade Católica De São Paulo, São Paulo, 2019.

MATEUS, Marta Elid Amorim. Um estudo sobre os conhecimentos necessários ao professor de Matemática para a exploração de noções concernentes às demonstrações e provas na Educação Básica. 2015. 269 f. Tese (Doutorado em Educação Matemática). Universidade Anhanguera de São Paulo, São Paulo, 2015.

MOREIRA, Plinio Cavalcanti; FERREIRA, Ana Cristina. O lugar da matemática na Licenciatura em Matemática. Bolema, v. 27, n. 47, p. 981-1005, dez. 2013.

MOURA, Rosimeire da Silva Rosa. Conhecimentos e práticas sobre o ensino de matemática expresso pelas professoras que atuam na educação infantil no município de Ribas do Rio Pardo/MS. 2016. 131 f. Dissertação (Mestrado em Educação). Universidade Estadual de Mato Grosso do Sul, Campo Grande, 2016.

NÓVOA, António Manuel Seixas Sampaio da. Desafios do Trabalho e Formação Docentes. 2017. (1h17m8s). Disponivel em: <https://www.youtube.com/watch?v=sYizAmj1rM\&list=PL2aXWIUwri9fKRPGAK3pukIP0vA9RjWZK\&index=4>. Acesso em: 02 de set. de 2020.

OLIVEIRA, Barbara Passadore de. O conceito de simetria na álgebra escolar: um estudo baseado na análise de documentos oficiais e manuais de professores. 2015. 78 f. Dissertação (Mestrado em Ensino e História das Ciências e da Matemática). Universidade Federal Do ABC, Santo André, 2015. 
OLIVEIRA, Felipe Augusto Pereira Vasconcelos Santos e. Analisando a mobilização de conhecimentos algébricos de professores de Educação Básica: o momento de preparação de aulas sobre equações. 2014. 161 f. Dissertação (Mestrado em Ensino e História das Ciências e da Matemática). Universidade Federal Do ABC, Santo André, 2014.

PEREIRA, Fernando Francisco. Conhecimentos mobilizados por graduandos e professores que ensinam matemática em um curso de formação sobre tarefas de análise da produção escrita. 2019. 124 f. Dissertação (Mestrado Profissional em Ensino de Matemática). Universidade Tecnológica Federal do Paraná, Londrina, 2019.

RIBEIRO, Rogerio Marques. Modelagem matemática e mobilização de conhecimentos didáticos matemáticos na formação continuada de professores dos anos iniciais. 2016. 263 f. Tese (Doutorado em Educação). Universidade Federal de São Carlos, São Carlos, 2016.

ROGERI, Norma Kerches de Oliveira. Conhecimentos de professores dos anos iniciais para o ensino dos números racionais em sua representação decimal. 2015. 289 f. Tese (Doutorado em Educação Matemática). Universidade Anhanguera de São Paulo, São Paulo, 2015.

ROLDÃO, Maria do Céu. Função docente: natureza e construção do conhecimento profissional. Revista Brasileira de Educação, v.12, n.34, p. 94-103, 2007.

SANTOS, Graca Luzia Dominguez. Um modelo teórico de matemática para o ensino do conceito de função. 2017. 165 f. Tese (Doutorado em Ensino, Filosofia E História Das Ciências). Universidade Federal da Bahia, Salvador, 2017.

SCHIFTER, D. Learning Mathematics for Teaching: From a Teachers' Seminar to the Classroom. Journal for Mathematics Teacher Education, 1(1), p. 55-87, 1998.

SERA, Eduardo Keidin. Conhecimentos de professores para o ensino da leitura e construção de gráficos estatísticos na educação básica. 2016. 216 f. Dissertação (Mestrado em Educação Matemática). Universidade Anhanguera de São Paulo, São Paulo, 2016.

SERRA, Rodrigo Donizete. O conhecimento matemático para o ensino e os "por quês" dos alunos. 2018. 104 f. Dissertação (Mestrado em Educação). Universidade Federal de São Carlos, Sorocaba, 2018.

SHULMAN, Lee Shulman. Those who understand: Knowledge growth in teaching. Educational Researcher, 15, 4-14, 1986.

SHULMAN, Lee Shulman. Knowledge and teaching: foundations of the new reform. Harvard Educational Review, v.57, n.1, p.1-22, 1987.

SILVA, Carolina Moura Brasil Carneiro da. Além da Zona de Conforto: Problematizando o Conhecimento de Matemática para o Ensino. 2016. 77 f. Dissertação (Mestrado em Ensino de Matemática). Universidade Federal do Rio de Janeiro, Rio de Janeiro, 2016.

SILVA, Regina de Lima. Conhecimentos matemáticos de professores dos anos iniciais do ensino fundamental: um estudo sobre o jogo da velha com figuras geométricas como recurso didático. 2017. 150 f. Dissertação (Mestrado em Educação Matemática e Tecnológica). Universidade Federal de Pernambuco, Recife, 2017. 
SILVA, Susana Maris Franca da. Formação de professores dos anos iniciais: uma investigação sobre os conhecimentos para o ensino de área e perímetro de figuras planas. 2016. $132 \mathrm{f}$. Dissertação (Mestrado em Educação Matemática). Universidade Anhanguera de São Paulo, São Paulo, 2016.

SILVA, Thais Helena Inglez. Conhecimento do professor de Matemática sobre equações: analisando o processo avaliativo sob o olhar de um modelo de perfil conceitual. 2015. $153 \mathrm{f}$. Dissertação (Mestrado em Ensino e História das Ciências e da Matemática). Universidade Federal do ABC, Santo André, 2015.

SOUZA, Debora da Silva. A formação do professor de Matemática: um estudo sobre 0 conhecimento pedagógico dos números racionais. 2015. 149 f. Dissertação (Mestrado em Ensino e História das Ciências e da Matemática). Universidade Federal do ABC, Santo André, 2015. 